Thorax (1974), 29, 559.

\title{
Rupture of the diaphragm
}

\author{
L.A.CHR IST I A NSEN ${ }^{1}, P$. S T A GE, \\ E. B ILLE BRAHE, and S. BERTELSEN \\ Department of Thoracic Surgery and the Department of Radiology, \\ Rigshospitalet, Copenhagen, Denmark
}

\begin{abstract}
Christiansen, L. A., Stage, P., Bille Brahe, E., and Bertelsen, S. (1974). Thorax, 29, 559-563. Rupture of the diaphragm. A 23-year series of 25 patients with rupture of the diaphragm is presented.

Symptoms, diagnostic procedures, and treatment are mentioned. A high index of suspicion of the diagnosis of ruptured diaphragm is most important in patients with a history of trauma.

Familiarity with the signs on the plain chest film is important. Furthermore, we advocate an additional examination, that is diagnostic pneumoperitoneum, in all cases of suspected rupture of the diaphragm.

If doubt still exists, we consider the final diagnostic procedure of choice to be exploratory thoracotomy until proof of the complete reliability of diagnostic pneumoperitoneum has been established.
\end{abstract}

The clinical manifestations of traumatic diaphragmatic hernia were described by Paré in 1580, and a torn left diaphragm was successfully repaired in 1899 by Walker (Andrus and Morton, 1970).

A traumatic lesion of the diaphragm is infrequent except in wartime but is seen with increasing frequency resulting from traffic accidents.

In the Anglo-Saxon literature from the period 1958-69, Hood (1971) collected 429 cases of rupture of the diaphragm. Seventy-eight patients died.

Hood concluded that the deaths reflect two facts: frequently the diagnosis of ruptured diaphragm is overlooked, and associated injuries in many patients result in death.

Lately, several patients have been admitted to the department of thoracic surgery with undiagnosed rupture of the diaphragm. To draw attention to this lesion we present our material compiled during the past 23 years. Symptoms, diagnosis, and operative procedures will be discussed. Especially we advocate the old but infrequently applied procedure of diagnostic pneumoperitoneum.

\section{PATIENTS}

Twenty-five patients were operated upon for ruptured diaphragm in the department of thoracic surgery, Rigshospitalet, during the period 1950-73.

1Address correspondence to: L. A. Christiansen, Department of Surgical Gastroenterology C, Rigshospitalet, Copenhagen, Denmark
The rupture occurred on the left side in 18 patients and on the right side in seven patients. The rupture was never bilateral. In 21 patients the associated injuries listed in Table I were seen-some combined. Twenty patients were males, five females, their ages ranging from 10 to 78 years. Traffic accidents caused the lesion in 17 patients; falls were responsible in three cases. The rupture originated from stab lesions in four patients, and from disruption of a surgical wound of the diaphragm in one patient only. In all, four patients died.

T A B L E I

TYPE OF ASSOCIATED INJURIES SEEN IN 21 PATIENTS

\begin{tabular}{l|c}
\hline \multicolumn{1}{|c|}{ Type of Injury } & No. of Patients \\
\hline Fracture (excepting thoracic cage) & 15 \\
Liver lesion & 3 \\
Spleen rupture & 1 \\
Digestive tract lesion & 4 \\
Cerebral lesion & 3 \\
\hline
\end{tabular}

DIAGNOSIS

In 12 patients the diagnosis was established during the first 10 post-traumatic days. In this group an elevated and blurred diaphragm, pleural effusion, lower lobe atelectasis, and a viscus in an abnormally high position seen in the chest film taken on admission confirmed the indication for thoracotomy. Excepting symptoms referable to 
the thorax, no subjective complaints were recorded in this group.

The diagnosis was established in the remaining 13 patients 10 days to four and a half years following the trauma. Among these patients seven complained of pain in the lower thorax radiating to the shoulder, epigastric pain, and dyspnoea. Three patients had no symptoms at all but were admitted exclusively because of the permanent abnormal findings in the chest radiograph.

CHEST RADIOGRAPH A preoperative film was available in 24 patients, since one patient with a stab wound was operated on immediately after admission.

All patients were examined in recumbent position, some in the upright position as well. The findings are presented in Table II.

T A B L E I I

CHEST X-RAY IN 24 PATIENTS WITH RUPTURE OF THE DIAPHRAGM

\begin{tabular}{l|c}
\hline \multicolumn{1}{c|}{ Abnormal Findings } & No. of Patients \\
\hline Apparently high cupola of the diaphragm & 22 \\
Gas-containing viscus in high position & 17 \\
Mediastinum displaced to opposite side & 15 \\
Atelectasis at the lung base & 21 \\
Pleural effusion & 16 \\
Fractures in thoracic cage & 10 \\
Pneumothorax & 1 \\
\hline
\end{tabular}

The lungs and diaphragmatic domes were normal in the film in one patient only, a young man with a stab wound, but in this patient an enlarged heart shadow demonstrated the presence of haemopericardium.

Barium meal or enema examinations were performed in seven patients to confirm an abnormally high position of the stomach or the bowels. In all these patients the abnormal position was confirmed, and in two patients a constriction of the stomach at the level of the rupture directly confirmed the diagnosis of herniation.

DIAGNOSTIC PNEUMOPERITONEUM Conventional chest radiographs and barium examinations do not demonstrate the diaphragmatic rupture itself. The differential diagnosis between a rupture and an elevation of the diaphragm may be difficult in some cases. By insufflation of air into the peritoneal cavity (diagnostic pneumoperitoneum) the diaphragm itself can always be localized, and if a pneumothorax results, the diagnosis of rupture is established.

With the aim of investigating the reliability of this examination we have performed diagnostic pneumoperitoneum, when possible, in all patients admitted during the last two years planned to be $\frac{\bar{\omega}}{\bar{n}}$ operated on for suspected rupture of the diaphragm.

Altogether nine patients were examined. In two कs patients a rupture of the diaphragm was diag- $\vec{\circ}$ nosed: one was left-sided (Fig. 1) and was con- $\overrightarrow{-}$ stantly diagnosed as a post-traumatic paralysis of $\tilde{O}^{\vec{\omega}}$ the diaphragm during four and a half years; the other was right-sided (Fig. 2), an acute rupture with herniation of the whole liver.

In seven patients we found an elevated intact is diaphragm by pneumoperitoneum (Figs 3 and 4). Three patients underwent no exploratory thoraco-o tomy because of age and an unconvincing clinical ${ }_{\triangle}^{\supset}$ picture. The four other patients had thoracotomyes performed, and the diagnosis established byo pneumoperitoneum was verified in all.

The technique for diagnostic pneumoperitoneum is simple. Using local anaesthesia, the peritoneum in the left hypogastric region is punc- $\overrightarrow{0}$ tured with a thin Teflon catheter fitted with an $\$$ inner metal needle (Longdwell $18 \mathrm{G}$ ). Duringo puncture saline is injected through the needle, and when resistance can no longer be felt the inner needle is removed and the Teflon catheter is. further advanced over a soft metal guide wire. $\frac{\mathrm{D}}{\mathrm{O}}$ Atmospheric air is insufflated until the patient expresses a feeling of diffuse pressure in the abdo-응 men, usually after $400 \mathrm{ml}$. The air is directed to the side of suspected rupture by changing the position of the patient. Chest films are taken in as upright a position as possible.

\section{TREATMENT}

A thoracotomy was performed and the rupture was sutured with double interrupted silk. If herniation was present the hernial contents were reduced. In two cases strangulation of the stomach 9 was present and resection of devitalized tissue was? performed. When a patient was admitted for emergency operation the intra-abdominal organs $\tilde{O}_{\sim}$ were visualized through the diaphragmatic rupture.

\section{DISCUSSION}

A tear of the diaphragm might be the result of blunt or penetrating thoracic or thoraco-? abdominal trauma. As recorded in other series (Bernatz, Burnside and Clagett, 1958; Asbury, $\frac{\vec{P}}{\circ}$ 1968), we also most frequently found the lesion on the left side. These authors state that the right $\stackrel{\mathbb{Q}}{\Omega}$ diaphragm is protected by the liver. Whether thiso is the true explanation or whether right-sided 


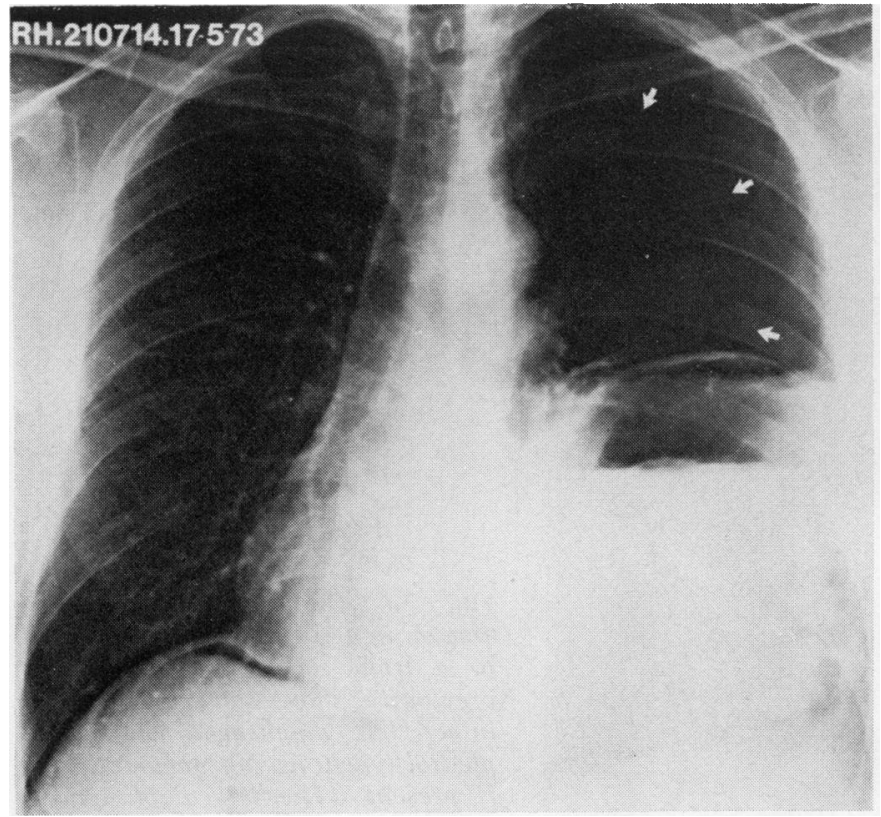

FIG. 1. Diagnostic pneumoperitoneum in a 43-year-old man exposed to a traffic accident four and a half years previously shows pneumothorax on the left side, indicating a rupture of the left diaphragm. (The lung border is marked by arrows.)

FIG. 2. Diagnostic pneumoperitoneum in a 38-year-old man with multiple lesions caused by a traffic accident nine days previously shows formation of a pneumothorax on the right side, indicating a rupture of the right diaphragm. (The lung border is marked by arrows.)

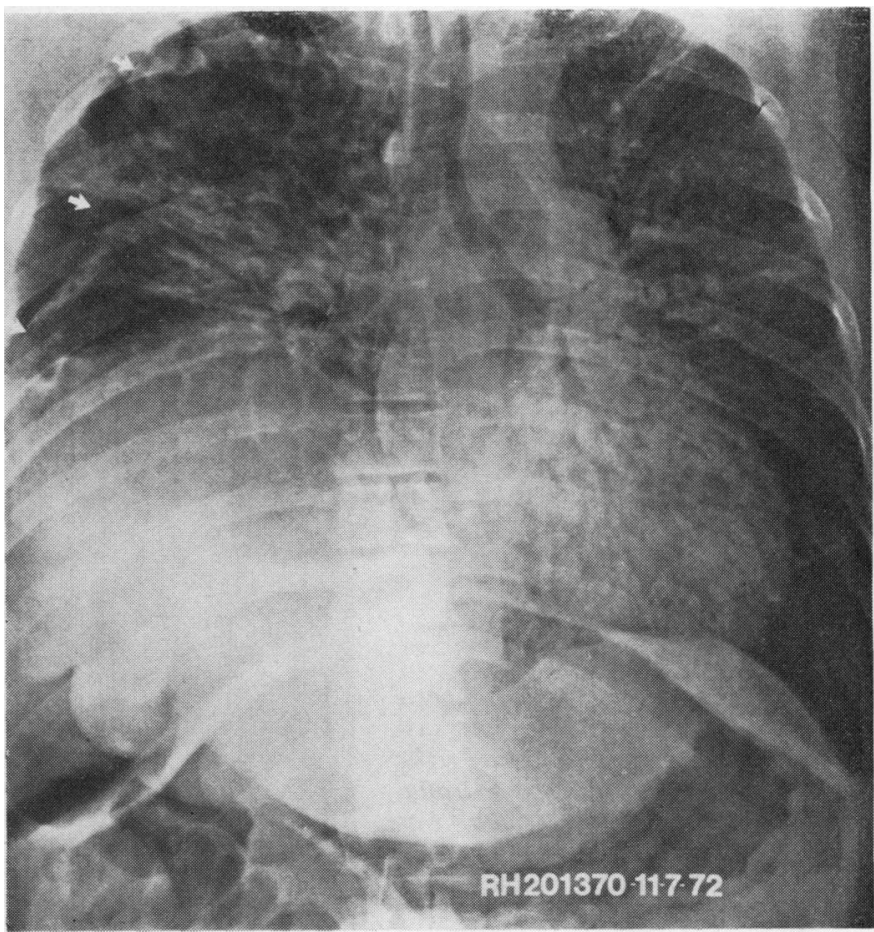




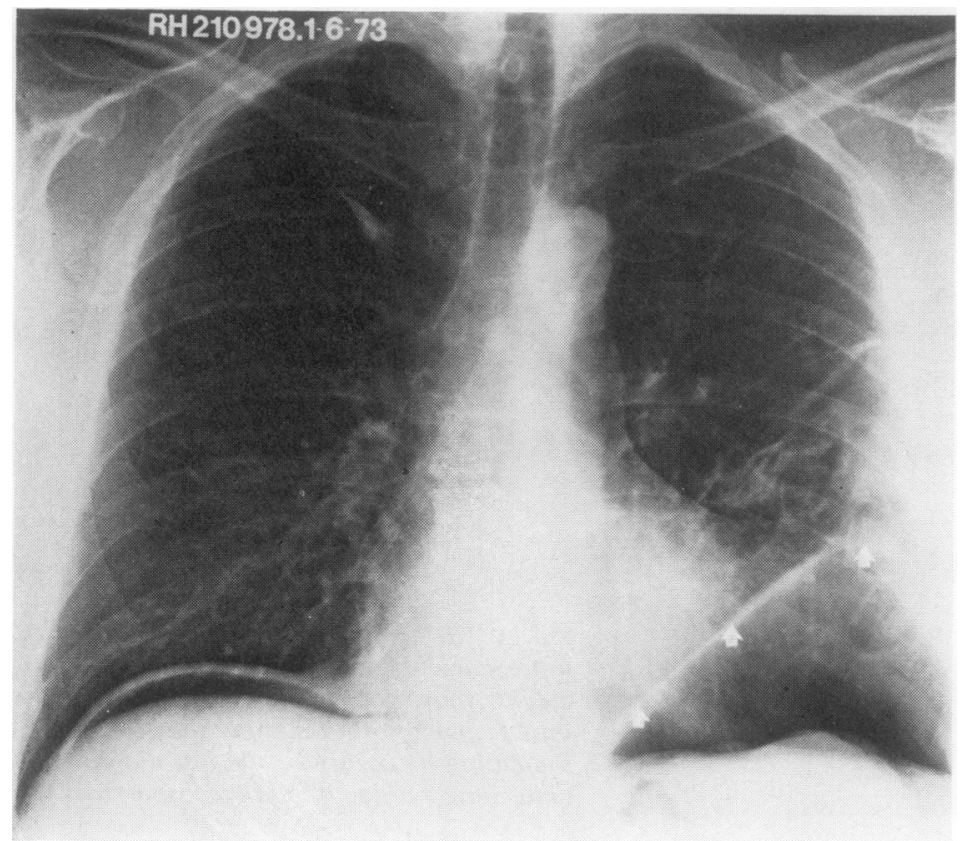

FIG. 3. Diagnostic pneumoperi- $\frac{\mathbb{D}}{\mathrm{C}}$ toneum in a 43-year-old man exposed 3 to a traffic accident four months previously shows an elevated but intact left diaphragm with basal $\overrightarrow{0}$ pleural reactions. No pneumothorax $\rightarrow$ is present. (The left diaphragm is marked by arrows.)

FIG. 4. Diagnostic pneumoperitoneum in a 57-year-old man suspected of rupture of the right diaphragm following a traffic accident shows an elevated but intact diaphragm. No pneumothorax is present. (The right diaphragm is marked by arrows.)

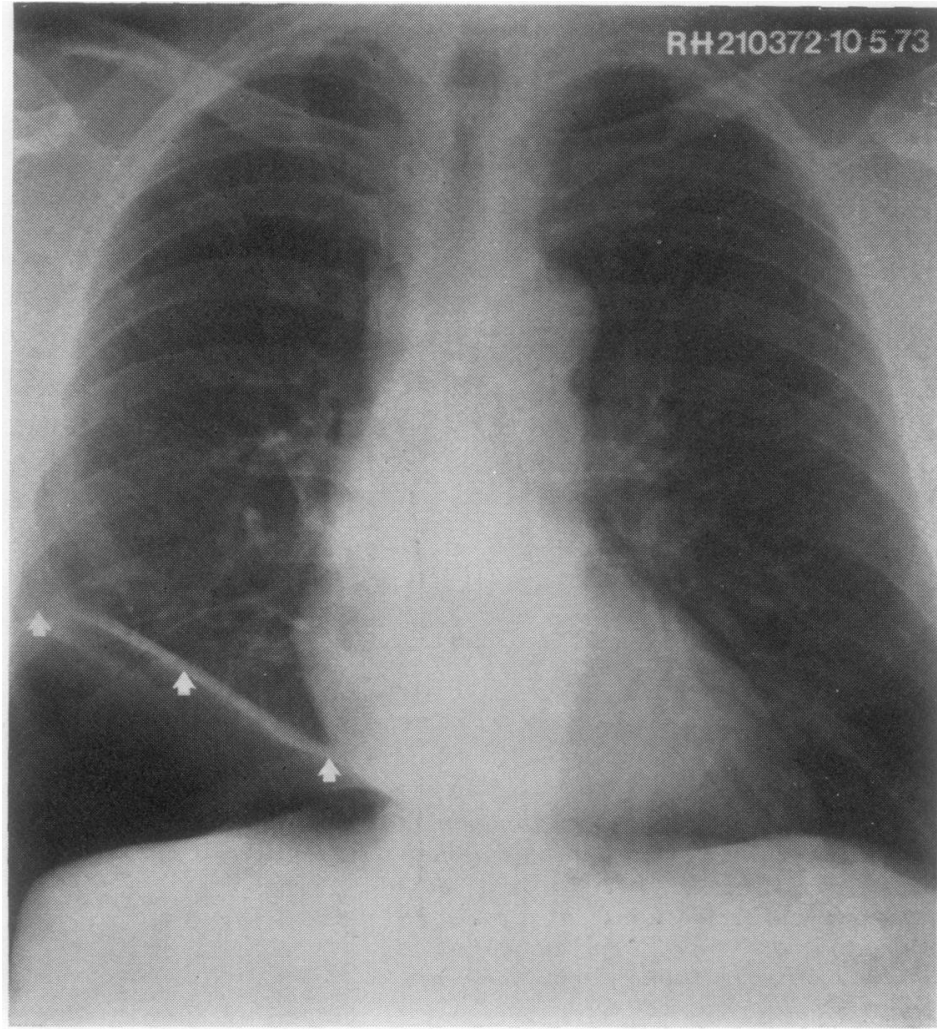


diaphragmatic lesions are most often overlooked cannot be elicited from this material.

By far the most common aetiology was a blunt trauma from traffic accidents, explaining the younger persons' predominance in our material.

In most cases diagnosed, an intra-abdominal viscus was herniated through the defect. In other cases a tear existed without initial herniation. Days or weeks later a hernia might result from the higher intra-abdominal pressure compared to that of the thorax (Andrus and Morton, 1970).

In our series four patients died. Three deaths were caused by associated injuries. One patient died two days after the trauma from a cerebral lesion, another, five days after trauma from a liver lesion. The third patient died four months postoperatively from intractable sepsis and confluent pneumonia. This patient was admitted with an overlooked strangulated incisional hernia of the diaphragm with perforation of the stomach causing empyema.

The last patient with a stab wound died six days postoperatively from a pulmonary embolus.

Our death rate is comparable to Hood's (1971).

Before diagnosis is discussed it should be emphasized that most important for the diagnosis of a diaphragmatic lesion is the high index of suspicion when a patient is admitted after injury (Bernatz et al., 1958; Asbury, 1968; Hill, 1972).

The complaints in uncomplicated rupture of the diaphragm are: lower thoracic pains explained by irritation of the diaphragm, and dyspnoea caused by compression atelectasis and/or effusion into the pleural cavity.

When incarceration and strangulation complicate the condition varying degrees of gastrointestinal obstruction with or without haematemesis might be present, depending upon which organ has been strangulated. Further complications consist of perforation, empyema, and sepsis.

The diagnosis is based largely upon radiographic examination. Keeping the diagnosis in mind, a chest film will give the diagnosis or at least the suspicion (Jensen and Lockwood, 1965).

In our series, a characteristic finding on the chest film seen in 22 patients was the apparently high position of the diaphragm which was in fact the upper border of a herniated organ, and this outline looked blurred due to pleural effusion.

Gas-containing viscus in an abnormally high position was recorded in 17 patients.

Shift of the mediastinum to the opposite side, seen in 15 patients, was caused by the herniated organs, pleural effusion or change of intrapleural pressure.

Diagnostic pneumoperitoneum, by which the diaphragm is directly visualized, is mentioned in the literature but not often employed (Kümmerle and Klöss, 1957; Meschan, 1966). The reason, presumably, is fear of air embolism and pneumothorax leading to respiratory collapse (Meschan, 1966).

From a theoretical point of view diagnostic pneumoperitoneum should be reliable in fresh diaphragmatic tears. In overlooked diaphragmatic ruptures adhesions from the herniated organs might be suspected of preventing air from reaching the pleural cavity. In such cases the distinct diaphragmatic cupola will not be seen.

In our material free passage of air from the peritoneal to the thoracic cavity was seen in a patient injured four and a half years prior to examination, although pronounced adhesions to the herniated organs were seen at operation.

We intend to continue the use of pneumoperitoneum in all cases when the diagnosis of a diaphragmatic tear is suspected but cannot be definitely established from a plain chest film.

Until the reliability of pneumoperitoneum is proved the indication for thoracotomy will be provided by the plain chest radiograph appearances.

\section{REFERENCES}

Andrus, C. H. and Morton, J. H. (1970). Rupture of the diaphragm after blunt trauma. American Journal of Surgery, 119, 686.

Asbury, G. F. (1968). Rupture of the diaphragm from blunt trauma. Archives of Surgery, 97, 801.

Bernatz, P. E., Burnside, A. F., and Clagett, D. T. (1958). Problem of the ruptured diaphragm. Journal of American Medical Association, 168, 877.

Hood, R. M. (1971). Traumatic diaphragmatic hernia. Annals of Thoracic Surgery, 12, 311.

Hill, L. D. (1972). Injuries of the diaphragm following blunt trauma. Surgical Clinics of North America, 52, 611.

Jensen, J. and Lockwood, K. (1965). Diaphragmarupturer. Ugeskrift for Lager, 127, 1406.

Kümmerle, F. and Klöss, J. (1957). Rechtsseitige traumatische Zwerchfellverletzungen mit Leberprolaps. Thoraxchirurgie, 5, 150.

Meschan, I. (1966). Roentgen Signs in Clinical Practice, p. 769. Saunders, Philadelphia.

Requests for reprints to: Dr. L. A. Christiansen, Department of Gastroenterology C, Rigshospitalet, Copenhagen, Denmark. 